Оригинальная статья/Original article

УДК 621.313

DOI: http://doi.org/10.20914/2310-1202-2017-3-31-34

Учет потерь на вихревые токи в обмотках электродвигателей с

полым якорем

Наталья В. Прибылова
Сергей А. ФИлонов
Екатерина Ю. Желтоухова 2

\title{
The count of losses by eddy currents in the windings of electric motors
} with hollow anchor

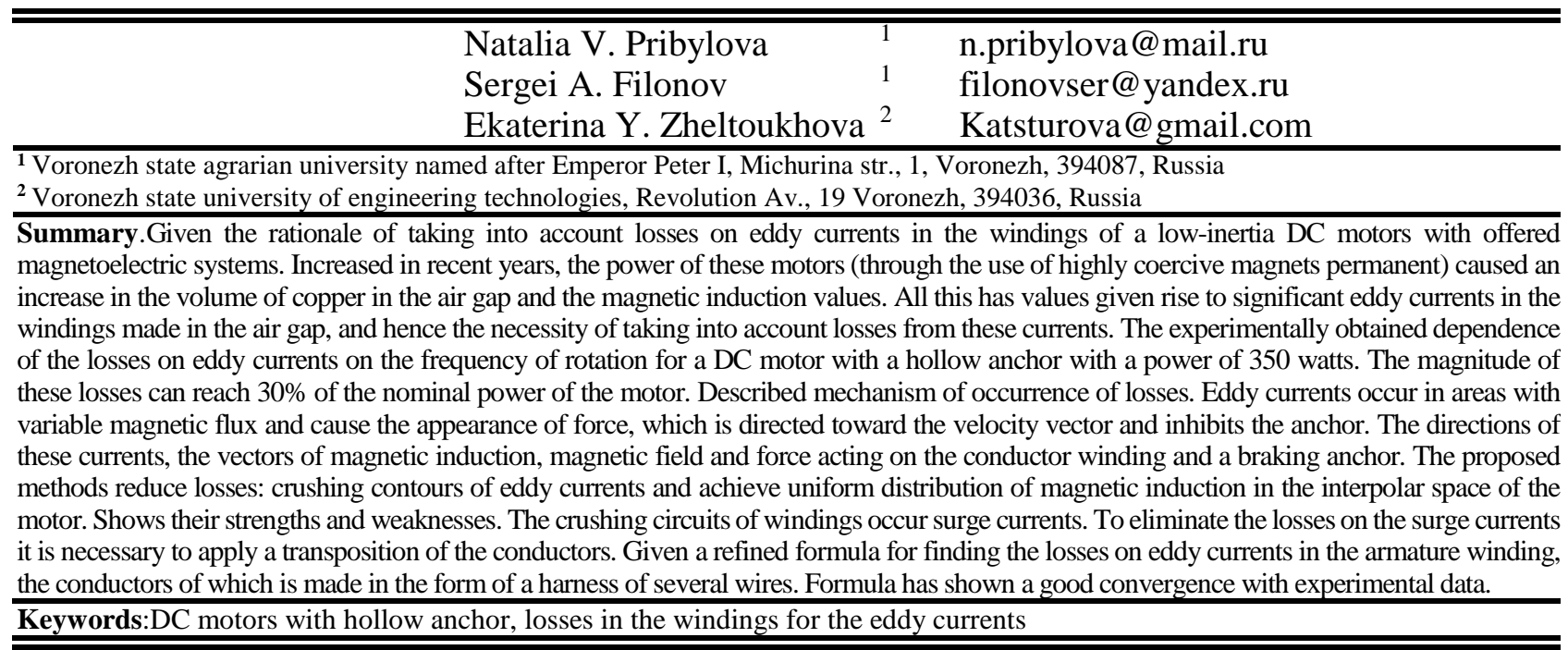

\section{Введение}

В настоящее время во многих устройствах и механизмах, используемых, в том числе и в сельскохозяйственном производстве, актуально применение малоинерционных электродвигателей постоянного тока с беспазовыми магнитоэлектрическими системами. В связи с ростом их габаритов и мощности, появилась необходимость учёта дополнительных потерь на вихревые токи

\section{Для цитирования}

Прибылова Н.В., Филонов С.А., Желтоухова Е.Ю. Учет потерь на вихревые токи в обмотках электродвигателей с полым якорем // Вестник ВГУИТ. 2017. Т. 79. № 3. С. 31-34. doi:10.20914/2310-12022017-3-31-34 в обмотках, вынесенных в воздушный зазор. Это связано с повышением рабочей частоты вращения, возрастанием числа пар полюсов магнитных систем, и, главное, с увеличением объема меди обмоток в относительно больших немагнитных воздушных зазорах с одновременным ростом магнитной индукции (из-за использования высококоэрцитивных магнитных материалов).

For citation

Pribylova N.V., Filonov S.A., Zheltouhova E.Ju. The count of losses by eddy currents in the windings of electric motors with hollow anchor. Vestnik VGUIT[Proceedings of VSUET]. 2017. vol. 79. no. 3. pp. 31-34. (in Russian). doi:10.20914/2310-1202-2017-3-31-34 
Целью работы является обоснование возникновения вихревых токов в обмотках электродвигателей с полым якорем и необходимости их учёта, а также определение оптимального способа для уменьшения потерь от вихревых токов.

До сих пор считалось, что в беспазовых компоновках такие потери необходимо учитывать при проектировании высокооборотных и весьма мощных преобразователей энергии в десятки киловатт и выше $[1,2]$ или более одного киловатта [3]. Однако, практика показывает, что значительные дополнительные потери на вихревые токи могут возникать и в обмотках машин меньшей мощности [4].

На рисунке 1 приведена экспериментально полученная зависимость потерь на вихревые токи $P_{\text {вихр.т. }} / P_{\text {ном }}$ от частоты вращения $\mathrm{n}$ для электродвигателя постоянного тока с полым якорем мощностью 350 Вт [5].

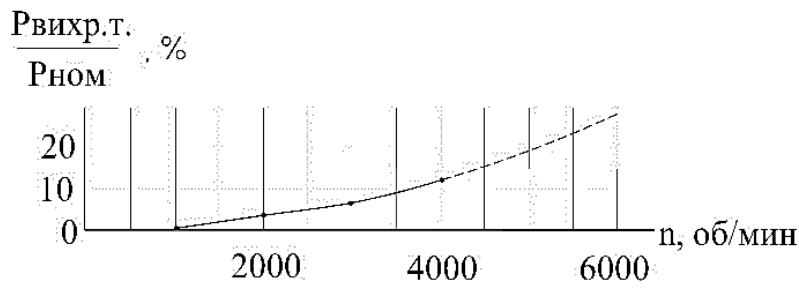

Рисунок 1. Добавочные потери на вихревые токи в процентах от номинальной мощности

Figure 1. Additional eddy current losses as a percentage of the rated power

Из зависимости, приведённой на рисунке 1 , видно, что потери мощности на вихревые токи имеют квадратичную зависимость от скорости вращения и при частоте вращения 6000 об/мин составляют величину около 100 Вт (пунктирная линия).

Рассмотрим подробнее механизм возникновения вихревых токов.

При вращении якоря с поднятыми щетками вихревые токи возникают в тех проводниках, в которых $d B / d t \neq 0$ (т. е. при большой крутизне возрастания магнитной индукции). На рисунке 2 показаны направления этих токов. Под северным полюсом слева они препятствуют возрастанию поля, а справа - поддерживают его исчезновение. Плотность вихревых токов

$$
j=-\frac{d H}{d y},
$$

где $H$ - напряженность магнитного поля; $x, y$ - оси координат.

В проводниках, находящихся в центре полюса, вихревые токи не возникают, если магнитная индукция неизменна. В этих проводниках наводится основная ЭДС, под действием которой ток в проводниках не протекает, так как при поднятых щетках все проводники можно условно считать разомкнутыми.

На рисунке 3 укрупненно показана одна проволока жгута обмотки якоря. Вихревые токи, направленные в одну сторону под северным полюсом и находящиеся в зоне с большими значениями магнитной индукции $B_{1}$, вызывают появление силы $F_{1}$, которая направлена навстречу вектору скорости и тормозит якорь. Плотность вихревых токов в зоне $B_{2}$ намного меньше и, соответственно, меньше сила $F_{2}$.

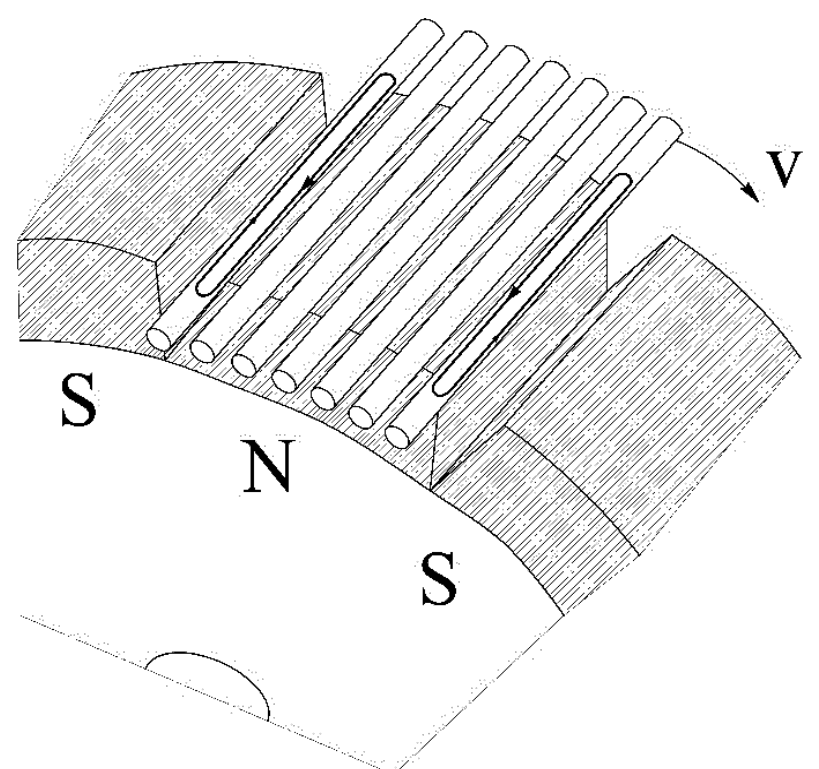

Рисунок 2. Распределение вихревых токов в обмотке полого якоря, равномерно вращающегося с поднятыми щетками в магнитном поле

Figure 2. The distribution of eddy currents in the winding of a hollow armature uniformly rotating with raised brushes in a magnetic field

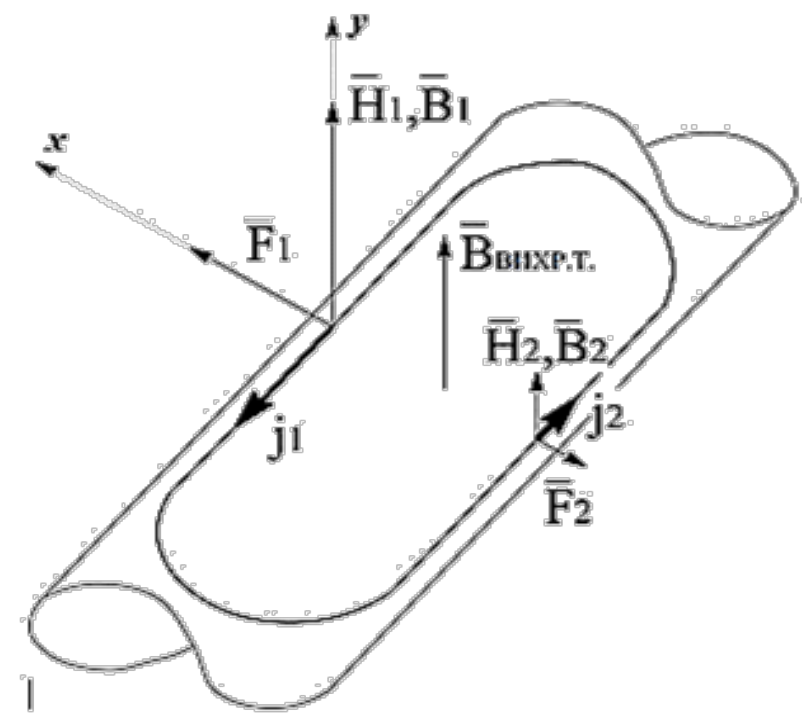

Рисунок 3. К определению сил, тормозящих якорь (под северным полюсом справа)

Figure 3. Determine forces that retard the anchor (under the north pole on the right) 
Существует несколько способов борьбы с потерями на вихревые токи:

1. Применяется дробление контуров вихревых токов, т. е. каждую секцию обмотки выполняют в виде жилы из нескольких изолированных проволок.

2. Достигается более плавное распределение магнитной индукции в межполюсном пространстве, без участков с большой крутизной.

В литературе $[6,7,8,9,10]$ приводятся формулы для определения потерь на вихревые токи, которые не представляется возможным использовать в нашем случае. Достаточно удовлетворительную сходимость с экспериментальными данными показала полученная нами уточненная формула.

Таким образом, потери на вихревые токи в обмотке полого якоря, проводники $N$ которого выполнены не в «навал», а в виде жгута (жилы) из нескольких проволок, уложенных параллельно образующей цилиндра («расчесанных») и трапецеидальной индукции в зазоре можно вычислить по следующей формуле

$$
P_{\text {ВихР }, T}=\frac{\pi^{3} N l_{a} d_{n p}^{4} a f^{2} B_{m}^{2}}{16 \rho}, \text { Вт }
$$

где $N$ - полное число проводников обмотки, выполненных в виде жгута (жилы) из нескольких проволок, параллельных образующей цилиндра якоря; $l_{a}$ - длина каждого проводника в пределах магнитного поля по оси якоря, м; $d_{n p}, a-$ диаметр круглой проволоки и число параллельных проволок в жгуте; $f$ - частота перемагничивания проводников $f=n p / 60 ; n$ - частота вращения,

\section{ЛИТЕРАТУРА}

1 Антонов А.Е., Киреев В.Г., Петухов И.С. Потери от уравнительных токов в многожильных обмотках беспазовых электрических машин // Техническая электродинамика. 2011. № 3. С. 33-39.

2 Васьковский Ю.Н., Титко А.И. Математическое моделирование вихревых токов и потерь в крайних пакетах сердечника статора турбогенератора //Техническая электродинамика. 2013. № 3. С. 50-56.

3 Данилина Э.М., Володин Г.И., Бреславец В.П. Потери энергии на вихревые токи в электромагнитных аппаратах вихревого слоя и способы их снижения // Известия высших учебных заведений. Электромеханика. 2014. № 1. C. 43-47.

4 Хомяк В.А., Прибылова Н.В. Анализ потерь в обмотке якоря электродвигателя постоянного тока // Энергия - XXI век. 2014. № 4 (88). С. 56-59.

5 Прибылова Н.В., Филонов С.А., Аксенов И.И. Потери на вихревые токи в обмотках электрических машин, вынесенных в воздушный зазор // В сборнике: Наука вчера, сегодня, завтра. Материалы научно-практической конференции. 2016. С. 236-239. об/мин; $p$ - число пар полюсов магнитной системы; $B_{m}$ - амплитудное значение магнитной индукции в зазоре, Тл, при $c / b=0,45 ; c, b-$ основания трапеции; $\rho-$ удельное электрическое сопротивление меди, $\rho=0,0173 \times 10^{-6} \mathrm{Oм} \cdot \mathrm{M}$.

При первом способе, кроме потерь на вихревые токи, определенных по формуле (2), в проволоках, соединенных между собой параллельно, на коллекторе, текут еще уравнительные токи. Это связано с тем, что все они находятся в разных магнитных условиях под полюсами, причем, чем больше проволок в жгуте, тем большее тормозное действие они оказывают на якорь. Для устранения потерь на уравнительные токи необходимо обязательно применять транспозицию, например - скручивание проволок перед намоткой секций, что увеличивает трудоемкость изготовления якоря.

При втором способе, в результате уменьшения магнитного потока в воздушном зазоре, ухудшаются удельные характеристики и параметры электродвигателя.

\section{Заключение}

В современных электродвигателях с полым якорем необходимо учитывать потери на вихревые токи в обмотках, вынесенных в воздушный зазор. Такие обмотки необходимо выполнять в виде жгутов из нескольких проволок, обязательно применяя транспозицию проводников. При трапецеидальном распределении магнитной индукции в воздушном зазоре эти потери рассчитываются по уточнённой формуле (2), которая показала хорошую сходимость с экспериментальными данными.

6 Макарчук А.В. Аналитический расчет потерь мощности от вихревых токов в роторах с постоянными магнитами // Техническая электродинамика. 2015. № 6. С. 41-45.

7 Афанасьев А.А. Расчет магнитных потерь электромеханических устройств // Электричество. 2016. № 6. C. 25-33.

$8 \mathrm{Li}$ Jian et al. Effects of MMF harmonics on rotor eddy-current losses for inner-rotor fractional slot axial flux permanent magnet synchronous machines // Magnetics, IEEE Transactions on 48.2. 2012. P. 839-842.

9 Makarchuk A. Eddy currents losses in a rotor of the high-speed generator with the permanent magnet excitation // Tekhnichna Elektrodynamika. 2013. № 1. P. 56-61.

10 Yamazaki, Katsumi, Fukushima Effect of eddycurrent loss reduction by magnet segmentation in synchronous motors with concentrated windings//Industry Applications, IEEE Transactions on 47.2. 2011. P. 779-788.

\section{REFERENCES}

1 Antonov A.E., Kireev V.G., Petukhov I.S. Loss from surge currents in the multiconductor windings of electrical machines offered. Tekhnicheskaya elektrodinamika [Technical electrodynamics] 2011. no. 3. pp. 33-39. (in Russian) 
2 Vaskovsky Yu. N., Titko A.I. Mathematical modeling of eddy currents and losses in the extreme packages stator core of a turbogenerator Tekhnicheskaya elektrodinamika [Technical electrodynamics] 2013. no. 3. pp. 50-56. (in Russian)

3 Danilina E. M., Volodin G. I., Breslavets V. P. The energy Loss due to eddy currents in electromagnetic apparatuses of a vortex layer and methods of their reduction. Izvestiya vuzov [News of higher educational institutions. Electrician] 2014. no. 1. pp. 43-47. (in Russian)

4 Homyak V.A., N.V. Pribylova Analysis of the loss in the armature winding of the electric motor DC. Energiya - XXI vek. [Energy - XXI century] 2014. no. 4 (88). pp. 56-59. (in Russian)

5 Pribylova N.V., Filonov S.A., Aksenov I.I. Losses on vortex currents in windings of electrical machines, made in the air gap. Nauka vchera, segodnya, zavtra [Science yesterday, today and tomorrow. Proceedings of scientific-practical conference] 2016. pp. 236-239. (in Russian)

\section{СВЕДЕНИЯ ОБ АВТОРАХ}

Наталья В. Прибылова к.т.н., доцент, кафедра электротехники и автоматики, Воронежский государственный аграрный университет имени императора Петра I, ул. Мичурина, 1, г. Воронеж, 394087, Россия,n.pribylova@mail.ru Сергей А. Филонов к.т.н., доцент, кафедра электротехники и автоматики, Воронежский государственный аграрный университет имени императора Петра I, ул. Мичурина, 1, г. Воронеж, 394087, Россия,filonovser@yandex.ru

Екатерина Ю. Желтоухова к.т.н., доцент, кафедра жиров, процессов и аппаратов химических и пищевых производств, Воронежский государственный университет инженерных технологий, пр-т Революции, 19, г. Воронеж, 394036, Россия,Katsturova@gmail.com

\section{КРИТЕРИЙ АВТОРСТВА}

Все авторы в равной степени принимали участие в написании рукописи и несут ответственность за плагиат

\section{КОНФЛИКТ ИНТЕРЕСОВ}

Авторы заявляют об отсутствии конфликта интересов.

ПОСТУПИЛА 11.05.2017

ПРИНЯТА В ПЕЧАТЬ 28.07.2017
6 Makarchuk V. A. Analytical calculation of power losses from eddy currents in the rotor with permanent magnets. Tekhnicheskaya elektrodinamika [Technical electrodynamics] 2015. no. 6. pp. 41-45. (in Russian)

7 Afanas'ev A. A. The calculation of magnetic losses in Electromechanical devices. Elektrichestvo [Electricity] 2016. no. 6. pp. 25-33. (in Russian)

$8 \mathrm{Li}$, Jian, et al. Effects of MMF harmonics on rotor eddy-current losses for inner-rotor fractional slot axial flux permanent magnet synchronous machines. Magnetics, IEEE Transactions on 48.2. 2012. pp. 839-842.

9 Makarchuk A. Eddy currents losses in a rotor of the high-speed generator with the permanent magnet excitation. Tekhnichna Elektrodynamika. 2013. no. 1. pp. 56-61.

10 Yamazaki, Katsumi, Fukushima Effect of eddycurrent loss reduction by magnet segmentation in synchronous motors with concentrated windings. Industry Applications, IEEE Transactions on 47.2. 2011. pp. 779-788.

\section{INFORMATION ABOUT AUTHORS}

Natalia V. Pribylova candidate of technical sciences, assistant professor, electrical engineering and automatics department, Voronezh state agrarian university named after Emperor Peter I, Michurina str., 1, Voronezh, 394087, Russia,n.pribylova@mail.ru

Sergei A. Filonov candidate of technical sciences, assistant professor, electrical engineering and automatics department, Voronezh state agrarian university named after Emperor Peter I, Michurina str., 1, Voronezh, 394087, Russia,filonovser@yandex.ru Ekaterina Y. Zheltoukhova candidate of technical sciences, assistant professor, fat, processes and devices of chemical and food industries department, Voronezh state university of engineering technologies, Revolution Av., 19 Voronezh, 394036, Russia,Katsturova@gmail.com

\section{CONTRIBUTION}

All authors equally took part in writing the manuscript and are responsible for plagiarism

\section{CONFLICT OF INTEREST}

The authors declare no conflict of interest.

RECEIVED 5.11.2017

ACCEPTED 7.28.2017 